\title{
Number of consulting medical institutions and risk of polypharmacy in community- dwelling older people under a healthcare system with free access: a cross-sectional study in Japan
}

Toshiki Suzuki ${ }^{1,2}$, Masao Iwagami ${ }^{2,3^{*}}$, Shota Hamada ${ }^{3,4}$, Tomoyuki Matsuda ${ }^{2,3,5}$ and Nanako Tamiyaa

\begin{abstract}
Background: Under the Japanese free access healthcare system, patients are allowed to consult multiple medical institutions (including clinics and hospitals for general or specialist consultation) without primary care referral. This potentially increases the risk of polypharmacy. We examined the association between the number of consulting medical institutions and polypharmacy under a healthcare system with free access.
\end{abstract}

Methods: Via a self-administered questionnaire, we identified people aged $\geq 65$ years with $\geq 1$ disease and $\geq 1$ consulting medical institution in a Japanese city in 2016. The exposure of interest was the number of consulting medical institutions $(1,2$, or $\geq 3)$ and the outcome was polypharmacy (use of $\geq 6$ types of drugs). We performed a multivariate logistic regression analysis, adjusting for age, sex, household economy, and the number and type of comorbidities. To minimize confounding effects, we also performed propensity-score-matched analysis, categorizing patients into two groups: 1 and $\geq 2$ consulting medical institutions.

Results: Of 993 eligible individuals (mean (standard deviation) age: 75.1 (6.5) years, men: 52.6\%), 15.7\% (156/993) showed polypharmacy. Proportions of polypharmacy were 9.7\% (50/516), 16.6\% (55/332), and 35.2\% (51/145) for people who consulted 1,2, and $\geq 3$ medical institutions, respectively. Relative to people who consulted 1 medical institution, adjusted odds ratios (95\% confidence intervals) for polypharmacy were $1.50(0.94-2.37)$ and 3.34 (1.985.65) for those who consulted 2 and $\geq 3$ medical institutions, respectively. In propensity score matching, of 516 and 477 patients who consulted 1 and $\geq 2$ medical institutions, 307 pairs were generated. The proportion of polypharmacy was $10.8 \%(33 / 307)$ and $17.3 \%(53 / 307)$, respectively $(P=0.020)$. The odds ratio for polypharmacy $(\geq 2$ vs. 1 consulting medical institution) was 1.73 (95\% confidence interval 1.09-2.76).

(Continued on next page)

\footnotetext{
* Correspondence: iwagami-tky@umin.ac.jp

${ }^{2}$ Department of Health Services Research, Faculty of Medicine, University of

Tsukuba, 1-1-1 Tenno-dai, Tsukuba, Ibaraki 305-8575, Japan

${ }^{3}$ Health Services Research and Development Center, University of Tsukuba,

1-1-1 Tenno-dai, Tsukuba, Ibaraki 305-8575, Japan

Full list of author information is available at the end of the article
} 


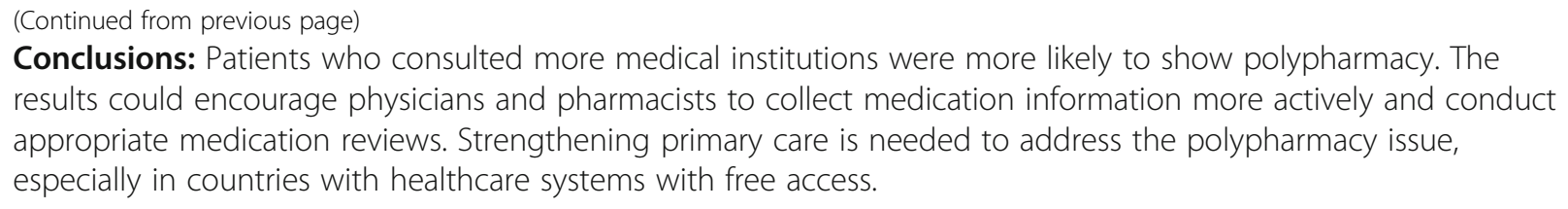

\section{Background}

Polypharmacy (i.e., use of multiple drugs) is known to be associated with an increased likelihood of adverse drug events, hospital admission, and mortality, particularly in older patients $[1-5]$. Under the public health insurance system with free access in Japan, patients choose which medical institutions they want to consult and may look up multiple medical institutions or specialists. Moreover, unlike other countries, there is no strict requirement to consult the hospitals of referral of primary care physicians. In Japan, doctors with any specialty can open a clinic for general care or specialist care in the community, and large hospitals can install both primary care and specialist care departments. Under this healthcare system, patients are allowed to visit any medical institution(s), including clinics and hospitals for general or specialist consultation. Although patients are advised to consult one physician regularly as a responsible primary care physician, a recent report suggested that the proportion of adults in Japan with a primary care physician was only $53.7 \%$ [6]. Moreover, older patients could be more likely to consult multiple medical institutions with different specialties because of multi-morbidity.

Previous studies have reported that the number and type of comorbidities experienced were associated with polypharmacy [7-10]. However, to the best of our knowledge, no studies have examined the association between the number of consulting medical institutions and the risk of polypharmacy, within the free access system, in older people living in the community. We hypothesized that consulting multiple medical institutions is an independent factor of polypharmacy and older patients who consulted multiple medical institutions are more likely to show polypharmacy, regardless of the number and type of comorbidities. Therefore, we aimed to examine an independent association between the number of consulting medical institutions and the risk of polypharmacy, using data from a cross-sectional survey conducted in a Japanese city.

\section{Methods}

\section{Data source}

We used data from a self-administered questionnaire survey conducted in December 2016, the original purpose of which was to formulate a welfare plan for the older citizens in Tsukuba City, Ibaraki, Japan, while the data have been secondarily used for research $[11,12]$. This questionnaire was performed by postal delivery and collection. Tsukuba City had a population of approximately 220,000, with approximately 42,000 people aged $\geq 65$ years (19.1\%), in 2016. As part of the survey, the questionnaire was sent randomly to 1500 residents aged 65-74 years and 1500 people aged $\geq 75$ years who lived at home and were not certified long-term care needs. The response rates were 50.6 and $53.2 \%$ for people aged $65-74$ and $\geq 75$ years, respectively. Therefore, the overall response rate was $51.9 \%(1557 / 3000)$. In addition, we restricted the analysis to people reporting having at least one disease, who were consulting at least one medical institution regularly $(n=993)$. This was because people with no disease and those currently visiting no medical institution for routine care are not at risk for polypharmacy. All patient identifiers were removed from the dataset. The present study was approved by the ethics committee of the University of Tsukuba (\#1166). Because of the anonymous nature of the data, the need for informed consent from individuals was waived.

\section{Independent variable, outcome, and covariates}

The independent variable was the number of consulting medical institutions (including clinics and hospitals for general or specialist consultation), based on responses to the question, "How many medical institutions do you currently visit for routine care?" The outcome of interest was polypharmacy, defined as the use of at least six drugs, following a study in which the risk of adverse drug events increased significantly in hospitalized older patients using six or more drugs [13]. We identified polypharmacy based on responses to the question, "How many types of prescribed medications do you currently take?"

As potential confounders, according to a directed acyclic graph (Additional file 1), we considered age, sex, household economy, and the number and type of comorbidities [7-10]. The household economy was based on responses to the subjective self-administered question, "How do you feel about your current daily situation economically?" Possible responses were as follows: "Very poor," "Poor," "Normal," "Rich," and "Very rich." The number and type of comorbidities were based on 
responses to the question, "Choose the diseases for which you are currently receiving treatment, from the following list (multiple answers possible)." The list of diseases was as follows: hypertension, stroke, heart disease, diabetes mellitus, dyslipidemia, respiratory disorder, gastrointestinal disorder, renal urologic disorder, musculoskeletal disorder, injury, malignancy, hematologic disease, depression, dementia, Parkinson's disease, ear disorder, and other diseases.

\section{Statistical analysis}

We described patient characteristics for 993 participants reporting having at least one disease and consulting at least one medical institution regularly. We determined the prevalence of polypharmacy according to the number of consulting medical institutions (one, two, and three or more).

We performed univariate and multivariate logistic regression analyses to estimate unadjusted and adjusted odds ratios with $95 \%$ confidence intervals (CIs) for the association between the number of consulting medical institutions and polypharmacy. To develop the multivariate logistic regression model, we selected variables significantly associated with polypharmacy in the univariate logistic regression models, at a threshold $p$-value of $<0.05$. As a sensitivity analysis, we added interaction terms among the comorbidities included in the multivariate logistic regression model.

Further, we performed propensity score matching as an additional analysis to better examine the independent association between the number of consulting medical institutions and polypharmacy [14]. In this analysis, we categorized the participants into two groups: those who consulted one and at least two medical institutions. The propensity score was estimated using the aforementioned covariates, and the $\mathrm{C}$ statistic was calculated to evaluate the goodness of fit. One-to-one nearestneighbor matching was performed based on estimated propensity scores for each participant, using a caliper within 0.20 standard deviations of the propensity score distribution. Of the propensity-score-matched patients, we compared the proportions of those who showed polypharmacy between groups via a chi-square test, followed by univariate logistic regression analysis of the association between the number of consulting medical institutions (at least two vs. one) and polypharmacy. As a sensitivity analysis, we repeated the propensity-scorematched analysis by categorizing patients into those who had consulted two or fewer and three or more medical institutions.

The significance level was set at $p<0.05$. All statistical analyses were performed using STATA version 14.2.

\section{Results}

Table 1 shows the baseline characteristics of the 993 eligible patients. Their mean age was 75.1 (standard deviation 6.5) years, and $52.6 \%$ were men. As shown in Fig. 1, patients who consulted higher numbers of medical institutions tended to be prescribed a higher number

Table 1 Characteristics of study participants

\begin{tabular}{|c|c|}
\hline \multirow[t]{2}{*}{ Factors } & $N=993$ \\
\hline & n (\%) \\
\hline Age (year old, mean $\pm S D$ ) & $75.1 \pm 6.5$ \\
\hline Sex (men) & $522(52.6)$ \\
\hline \multicolumn{2}{|c|}{ The number of consulting medical institutions } \\
\hline 1 & $516(52.0)$ \\
\hline 2 & $332(33.4)$ \\
\hline$\geq 3$ & $145(14.6)$ \\
\hline \multicolumn{2}{|l|}{ Household economy } \\
\hline Very poor & $57(5.9)$ \\
\hline Poor & $198(20.5)$ \\
\hline Normal & $633(65.4)$ \\
\hline Rich & $65(6.7)$ \\
\hline Very rich & $15(1.6)$ \\
\hline Not answered & $25(2.5)$ \\
\hline \multicolumn{2}{|l|}{ Comorbidities } \\
\hline Hypertension & $559(56.3)$ \\
\hline Stroke & $33(3.3)$ \\
\hline Heart disease & $132(13.3)$ \\
\hline Diabetes mellitus & $179(18.0)$ \\
\hline Dyslipidemia & $157(15.8)$ \\
\hline Respiratory disorder & $62(6.2)$ \\
\hline Gastrointestinal disorder & $84(8.5)$ \\
\hline Renal urologic disorder & $106(10.7)$ \\
\hline Musculoskeletal disorder & $103(10.4)$ \\
\hline Injury & $21(2.1)$ \\
\hline Malignancy & $49(4.9)$ \\
\hline Hematologic disease & $18(1.8)$ \\
\hline Depression & $11(1.1)$ \\
\hline Dementia & $11(1.1)$ \\
\hline Parkinson's disease & $1(0.1)$ \\
\hline Ear disorder & $54(5.4)$ \\
\hline Other & 108 (10.9) \\
\hline
\end{tabular}

Number of comorbidities

1

2

$447(45.0)$

$325(32.7)$

$133(13.4)$

$67(6.7)$

$21(2.1)$ 


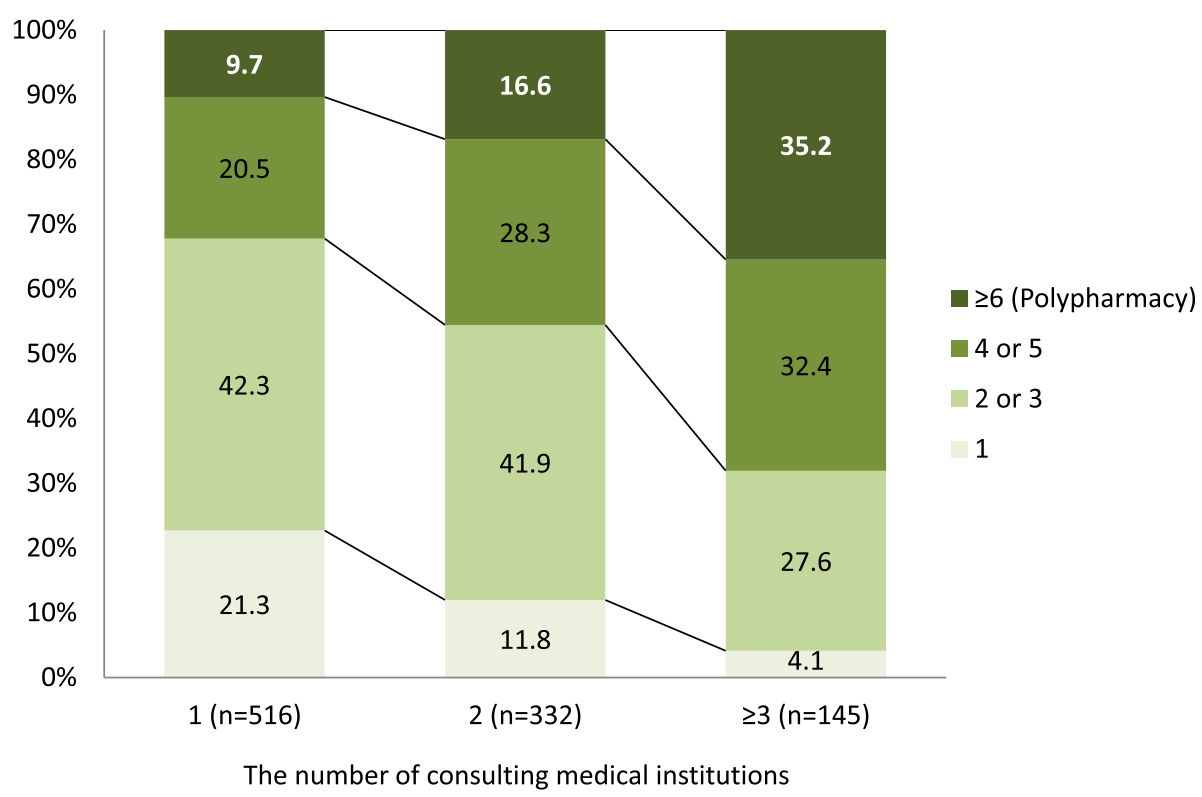

Fig. 1 Number of prescribed drugs according to number of consulting medical institutions

of drugs, relative to those who consulted fewer institutions.

In the univariate analysis, in comparison with participants who consulted one medical institution, the unadjusted odds ratios (95\% CIs) for polypharmacy were $1.85(1.23-2.79)$ and $5.06(3.23-7.92)$ for those who consulted two and three or more medical institutions, respectively (Table 2). In the multivariate analysis, the association was attenuated, but an association between the number of consulting medical institutions and polypharmacy remained; adjusted odds ratios (95\% CIs) were $1.50(0.94-2.37)$ and $3.34(1.98-5.65)$ for those who consulted two and three or more medical institutions, respectively. In the sensitivity analysis that included interaction terms among the comorbidities in the multivariate logistic regression model (i.e., heart diseases, diabetes mellitus, gastrointestinal disorders, renal urologic disorders, and musculoskeletal disorders), the adjusted odds ratios (95\% CIs) were $1.57(0.98-2.50)$ and 3.63 (2.11-6.24) for those who consulted two and three or more medical institutions, respectively.

As shown in Fig. 2, of the 516 and 477 participants who consulted one and at least two medical institutions, 307 propensity score-matched pairs were generated, with a $\mathrm{C}$ statistic of 0.716 . Following propensity score matching, the baseline patient characteristics were well balanced between groups (Table 3). The proportions of patients who showed polypharmacy were $10.8 \%(33 / 307)$ and $17.3 \%(53 / 307)$ in matched patients who had consulted one and at least two medical institutions $(P=0.020)$. Patients who consulted two or more medical institutions were more likely to show polypharmacy relative to matched patients who consulted one medical institution, with an odds ratio of 1.73 (95\% CI, 1.09-2.76).

In the sensitivity analysis, propensity matching was conducted for participants who consulted two or fewer and three or more medical institutions, and the resultant C statistic was 0.757 (Additional file 2 and Additional file 3). The proportions of patients who showed polypharmacy were $19.7 \%(26 / 132)$ and $34.4 \%(46 / 132)$ in matched patients who consulted two or fewer and three or more medical institutions $(P=0.006)$, with an odds ratio of 2.18 (95\% CI, 1.25-3.81).

\section{Discussion}

Free access to medical institutions is granted under the existing healthcare system in Japan; therefore, physicians do not always know which medical institutions patients have consulted or which drugs they have been prescribed, although patients are encouraged to consult specialists through referrals from primary care physicians. The current results showed that patients who consulted a high number of medical institutions tended to be at risk of polypharmacy. It is difficult for physicians to check and control the number of drugs prescribed by other physicians, particularly those at other medical institutions. The same is true of pharmacists. A likely explanation for the present results is that the number of opportunities to review and deprescribe some medications, if appropriate, was insufficient because physicians could not have known about or controlled all drugs prescribed by other physicians. In Japan, electronic medical records are available within the same institution. Thus, 
Table 2 Results of univariate and multivariate logistic regression analyses for polypharmacy

\begin{tabular}{|c|c|c|}
\hline & Unadjusted OR (95\% Cl) & Adjusted OR (95\% Cl) \\
\hline \multicolumn{3}{|c|}{ The number of consulting medical institutions } \\
\hline 1 & 1 (reference) & 1 (reference) \\
\hline 2 & $1.85(1.23-2.79)$ & $1.50(0.94-2.37)$ \\
\hline$\geq 3$ & $5.06(3.23-7.92)$ & $3.34(1.98-5.65)$ \\
\hline Age (every 1 year old increase) & $1.07(1.04-1.10)$ & $1.07(1.03-1.10)$ \\
\hline \multicolumn{3}{|l|}{ Sex } \\
\hline Women & 1 (reference) & 1 (reference) \\
\hline Men & $1.81(1.27-2.59)$ & $1.98(1.26-3.10)$ \\
\hline \multicolumn{3}{|l|}{ Household economy ${ }^{a}$} \\
\hline Very poor & $1.94(1.02-3.69)$ & $1.53(0.72-3.26)$ \\
\hline Poor & $1.56(1.03-2.34)$ & $1.50(0.95-2.38)$ \\
\hline Normal & 1 (reference) & 1 (reference) \\
\hline Rich $^{b}$ & $0.66(0.31-1.42)$ & $0.73(0.32-1.66)$ \\
\hline \multicolumn{3}{|l|}{ Very rich ${ }^{b}$} \\
\hline \multicolumn{3}{|l|}{ Comorbidities } \\
\hline Hypertension & $1.21(0.86-1.72)$ & \\
\hline Stroke & $1.20(0.49-2.96)$ & \\
\hline Heart disease & $3.81(2.53-5.74)$ & $2.89(1.75-4.76)$ \\
\hline Diabetes mellitus & $2.49(1.69-3.67)$ & $2.49(1.54-4.02)$ \\
\hline Dyslipidemia & $1.20(0.77-1.88)$ & \\
\hline Respiratory disorder & $1.79(0.97-3.29)$ & \\
\hline Gastrointestinal disorder & $2.35(1.42-3.91)$ & $1.71(0.92-3.19)$ \\
\hline Renal urologic disorder & $2.25(1.41-3.59)$ & $1.04(0.56-1.92)$ \\
\hline Musculoskeletal disorder & $2.35(1.47-3.76)$ & $2.44(1.34-4.47)$ \\
\hline Injury & $2.19(0.84-5.74)$ & \\
\hline Malignancy & $1.80(0.92-3.54)$ & \\
\hline Hematologic disease & $1.55(0.50-4.76)$ & \\
\hline Depression & $2.03(0.53-7.74)$ & \\
\hline Dementia & $3.12(0.90-10.79)$ & \\
\hline Parkinson's disease & Not available & \\
\hline Ear disorder & $1.08(0.52-2.25)$ & \\
\hline Other & $1.08(0.63-1.85)$ & \\
\hline \multicolumn{3}{|l|}{ The number of comorbidities } \\
\hline 1 & 1 (reference) & 1 (reference) \\
\hline 2 & $2.41(1.51-3.85)$ & $1.52(0.90-2.55)$ \\
\hline 3 & $4.28(2.51-7.29)$ & $1.58(0.83-2.99)$ \\
\hline 4 & $9.31(5.09-17.04)$ & $2.59(1.24-5.44)$ \\
\hline$\geq 5$ & 17.30 (6.78-44.09) & $2.24(0.68-7.43)$ \\
\hline
\end{tabular}

Cl confidence interval, $O R$ odds ratio

${ }^{\mathrm{a}} 25$ people with missing data were not included in the multivariate logistic regression analyses

${ }^{b}$ People in the rich and very rich categories were grouped because of the small number of participants

even if a patient consulted several specialists at the same medical institution, it would be easy for specialists at the same institution to check and control patients' prescribed drugs. However, electronic medical records cannot be shared by different institutions, meaning that physicians at different institutions cannot see what drugs have been prescribed by other physicians in different institutions. Therefore, patients visiting several institutions 


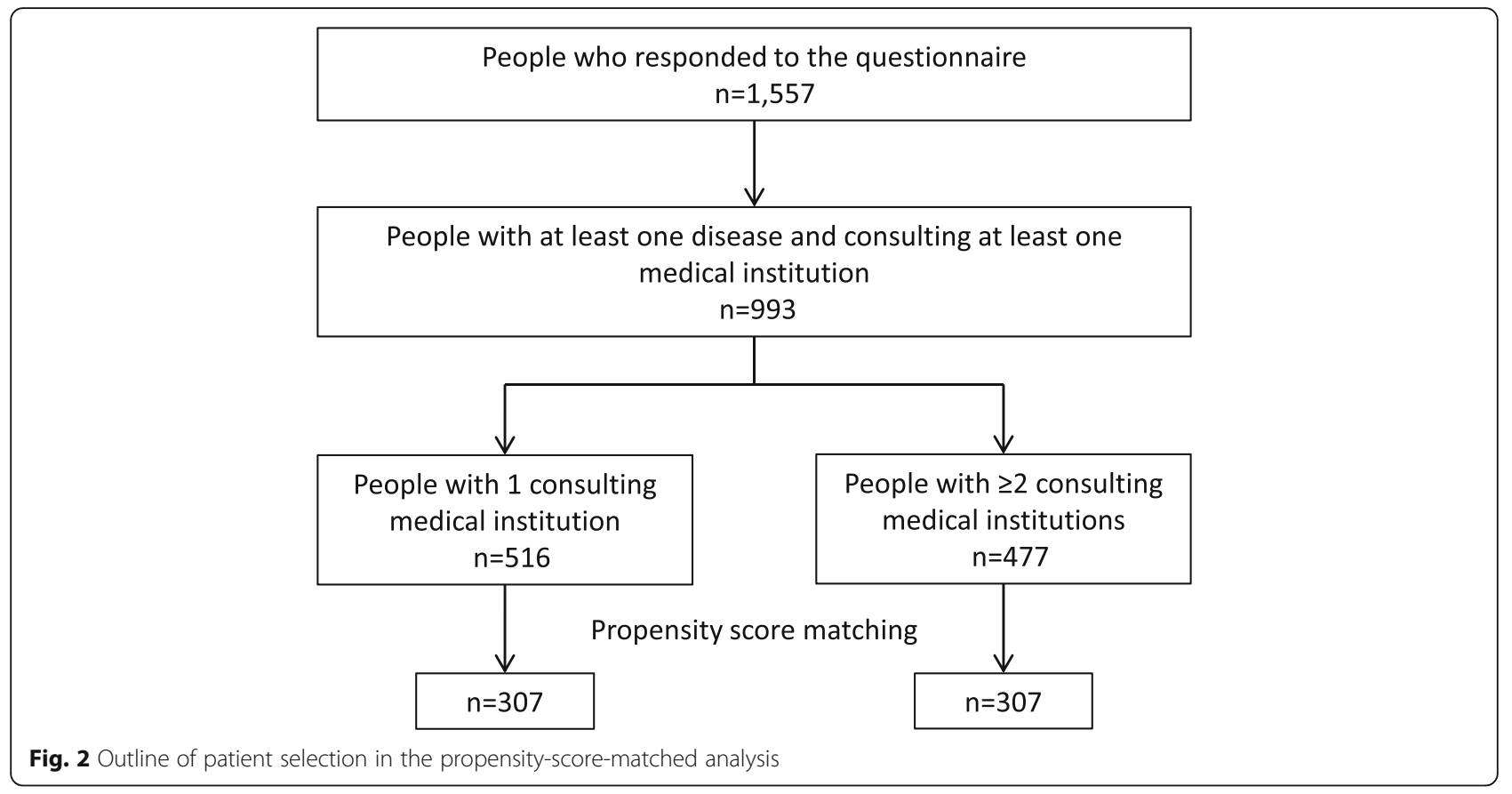

are more likely to be exposed to the risk of polypharmacy than patients consulting several specialists at the same institution, even if the number of consulting physicians is the same. According to previous studies conducted in Spain, the number of different specialists to which the patients were referred by their family physicians was significantly associated with adverse drug events and the high number of prescribers was a strong predictor of polypharmacy $[15,16]$. Although the healthcare system in Spain is different from Japan, these previous results are consistent with our findings.

Polypharmacy has been associated with the number and type of comorbidities [7-10]. Older patients with higher numbers of comorbidities were more likely to show polypharmacy $[7,8]$. A previous study reported a strong correlation between the number of diagnosed disorders and prescribed drugs [17]. Moreover, the likelihood of the occurrence of polypharmacy has been found to differ according to the type and combination of diagnosed disorders $[7,9,10]$. In addition, previous studies have shown that disorders associated with polypharmacy included cardiovascular disorder, an endocrine disorder, gastrointestinal disorder, urologic disorder, metabolic disease, frequent urination, and insomnia $[7,9]$. The results of our logistic regression analysis showed that heart disease, diabetes mellitus, gastrointestinal disorder, renal urologic disorder, and musculoskeletal disorder were associated with polypharmacy. This result was consistent with those of previous studies [7, 9]. One study demonstrated that patients with comorbidity combinations involving chronic kidney disease with osteoporosis, congestive heart failure with osteoporosis, chronic kidney disease with arthritis, and congestive heart failure with arthritis showed particularly high prevalence rates for polypharmacy [10]. Therefore, in the current study, adjustment for the number and type of comorbidities was essential to examine the independent association between polypharmacy and the number of consulting medical institutions. Our results suggest that men are more likely to show polypharmacy than women. This result might be caused by factors not included in our multivariate logistic regression analysis, such as the severity of comorbidities. A previous study conducted in Malaysia also showed that men were included in the significant risk factors [7], which is consistent with our findings. However, some studies reported that women were more likely to have polypharmacy $[10,18]$. The association between sex and polypharmacy remains controversial.

The study was subject to several limitations. First, the data analyzed in the study were collected using a questionnaire in a Japanese city, and the response rate was $51.9 \%$. Therefore, the generalizability of the results to other populations may be limited. We could not determine the characteristics of those who did not respond to the questionnaire due to the lack of relevant data. We anticipated that more health-conscious people would have been more likely to respond to the questionnaire. In our study, the proportion of polypharmacy (15.7\%) was lower than in the statistics from the Ministry of Health, Labour and Welfare, in Japan. The percentages of polypharmacy (defined as using five or more prescribed drugs) among people aged $65-74$ years and $\geq 75$ years in Japan as a whole were 28.0 and $41.1 \%$, respectively, in 2016 [19]. A previous study reported that the 
Table 3 Characteristics of participants before and after propensity score matching

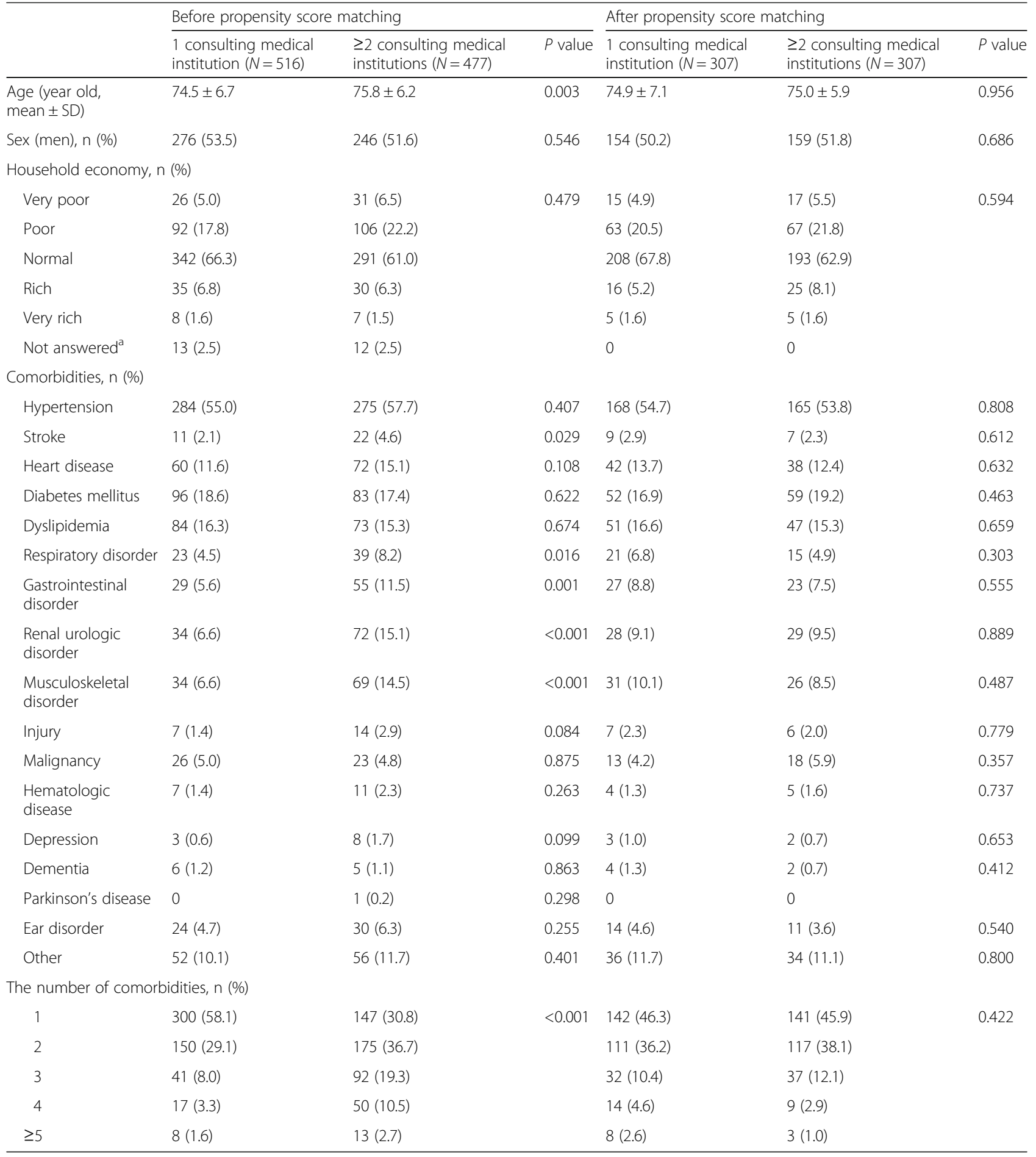

$S D$ standard deviation

${ }^{\mathrm{a}} 25$ people with missing data were not included in the propensity-score-matched analysis

proportion of polypharmacy (defined as using six or more prescribed medications) among residents aged $\geq 65$ years in an urban community in Tokyo was $28.0 \%$ [20]. The discrepancy is most likely explained by the fact that our study population consisted of healthier people without long-term care needs certification living at home. Thus, our results are not generalizable to people with long-term care needs certification. Further, the results of propensity score matching could be generalizable only to those in the range of propensity 
scores included in the paired analysis. Second, misclassification is likely to occur because the data were selfreported. We defined the independent variable (i.e., the number of consulting medical institutions) and outcome (i.e., polypharmacy) based on self-administered questionnaire items. If patients who consulted multiple medical institutions were more likely to overreport the number of drugs they were using, the results could have been overestimated. Third, although we adjusted for the number and type of comorbidities (i.e., important confounding factors according to previous studies [710]) in the multivariate logistic regression and subsequent propensity score analyses, residual confounding effects cannot be excluded entirely, as it was an observational study. For example, we were unable to obtain information regarding the severity of comorbid conditions. Patients with severe comorbidities could have been more likely to consult multiple medical institutions and receive higher numbers of prescriptions. Finally, we were unable to obtain details of prescriptions or the types of medication prescribed to patients. We could not judge the appropriateness of the drugs prescribed, because patients' prescription content, especially that regarding potentially inappropriate medication, was not taken into consideration in the study. Some patients might have required six or more drugs because of their complex conditions (i.e., appropriate polypharmacy). Further studies are needed, in which patients' prescription contents, especially regarding potentially inappropriate medications, are considered to evaluate the quality of polypharmacy. Despite its limitations, this study demonstrated that patients with a high number of consulting medical institutions tended to be exposed to a risk of polypharmacy.

\section{Conclusions}

The results of the study showed that patients who consulted a higher number of medical institutions were more likely to show polypharmacy, regardless of the number or type of comorbidities, relative to those who consulted fewer institutions. The results could encourage physicians and pharmacists to collect medication information more actively and conduct appropriate medication reviews. A responsible primary care physician who coordinates and reviews patients' medications is essential, especially for patients with multimorbidity, under healthcare systems with free access to medical institutions, including Japan. Strengthening primary care is necessary to address the polypharmacy issue. This study could not assess the appropriateness of the prescribed drugs. However, our results could contribute to identify the actions aimed at minimizing the polypharmacy issue under healthcare systems with free access.

\section{Supplementary information}

Supplementary information accompanies this paper at https://doi.org/10. 1186/s12913-020-05205-6.

Additional file 1. Directed acyclic graph.

Additional file 2. Outline of patient selection in the sensitivity analysis: Propensity score matching between patients who consulted two or fewer and three or more medical institutions.

Additional file 3. Characteristics of patients before and after propensity score matching in the sensitivity analysis: Propensity score matching between patients who consulted two or fewer and three or more medical institutions.

\section{Abbreviations}

Cl: Confidence Interval; SD: Standard Deviation

\section{Acknowledgments}

We would like to thank Editage (https://www.editage.jp/) for English language editing.

\section{Authors' contributions}

TS: conception and design, analysis, interpretation of data, drafting the article, revising the article critically for important intellectual content, and final approval of the manuscript. Ml: conception and design, analysis, interpretation of data, revising the article critically for important intellectual content, and final approval of the manuscript. SH: conception and design, interpretation of data, revising the article critically for important intellectual content, and final approval of the manuscript. TM: collection and

interpretation of data, revising the article critically for important intellectual content, and final approval of the manuscript. NT: conception and design, collection and interpretation of data, revising the article critically for important intellectual content, and final approval of the manuscript.

\section{Funding}

This study was supported by a grant-in-aid from the Ministry of Health, Labour and Welfare; Health and Labour Sciences Research Grant, Japan; Research on Policy Planning and Evaluation (H28-seisaku-ippan-003) and a grant-in-aid from the Ministry of Health, Labour and Welfare; Health, Labour and Welfare Policy Research Grant, Japan; (19AA2007). The funder played no particular role in conducting the study, but paid for English language editing and would pay for the journal acceptance fee.

\section{Availability of data and materials}

The data that support the findings of this study are available from the local government of Tsukuba City, Ibaraki, Japan, but restrictions apply to the availability of these data, which were used under administrative permissions for the current study, and so are not publicly available.

\section{Ethics approval and consent to participate}

All participants' identifiers were removed from the dataset. The present study was approved by the ethics committee of the University of Tsukuba (\#1166). Because of the anonymous nature of the data, informed consent from individual participants was waived. Administrative permissions by Tsukuba city, Ibaraki, Japan were acquired by our team to access the data used in our research.

\section{Consent for publication}

Not applicable.

\section{Competing interests}

Nanako Tamiya is Editorial Board Member.

\section{Author details}

${ }^{1}$ School of Medicine, University of Tsukuba, 1-1-1 Tenno-dai, Tsukuba, Ibaraki 305-8575, Japan. ${ }^{2}$ Department of Health Services Research, Faculty of 
Medicine, University of Tsukuba, 1-1-1 Tenno-dai, Tsukuba, Ibaraki 305-8575, Japan. ${ }^{3}$ Health Services Research and Development Center, University of Tsukuba, 1-1-1 Tenno-dai, Tsukuba, Ibaraki 305-8575, Japan. ${ }^{4}$ Research Department, Institute for Health Economics and Policy, Association for Health Economics Research and Social Insurance and Welfare, No.11 Toyo-Kaiji Bldg, 1-5-11 Nishi-Shimbashi, Minato-ku, Tokyo 105-0003, Japan. ${ }^{5}$ Department of Physical Therapy, School of Medical Health, Ibaraki Prefectural University of Health Sciences, 4669-2 Ami, Amimachi, Inashikigun, Ibaraki 300-0394, Japan.

Received: 23 October 2019 Accepted: 12 April 2020

Published online: 26 April 2020

\section{References}

1. Abe T, Tamiya N, Kitahara T, Tokuda Y. Polypharmacy as a risk factor for hospital admission among ambulance-transported old-old patients. Acute Med Surg. 2016;3(2):107-13.

2. Field TS, Gurwitz JH, Avorn J, et al. Risk factors for adverse drug events among nursing home residents. Arch Intern Med. 2001;161(13):1629-34.

3. Suzuki Y, Sakakibara M, Shiraishi N, Hirose T, Akishita M, Kuzuya M. Prescription of potentially inappropriate medications to older adults. A nationwide survey at dispensing pharmacies in Japan. Arch Gerontol Geriatr. 2018;77:8-12.

4. Onder G, Liperoti R, Foebel A, et al. Polypharmacy and mortality among nursing home residents with advanced cognitive impairment: Results from the Shelter Study. J Am Med Dir Assoc. 2013;14(6):450 e7-450.e12.

5. Fushiki Y, Kinoshita K, Tokuda Y. Polypharmacy and adverse drug events leading to acute care hospitalization in Japanese elderly. Gen Med. 2014:15(2):110-6.

6. Japan Medical Association Research Institute. Working paper. 2014. Available from URL: http://www.jmari.med.or.jp/download/WP331.pdf (in Japanese).

7. Lim LM, McStea M, Chung WW, et al. Prevalence, risk factors and health outcomes associated with polypharmacy among urban community-dwelling older adults in multi-ethnic Malaysia. PLoS One. 2017;12(3):e0173466.

8. Kojima T, Shimada K, Terada A, et al. Association between polypharmacy and multiple uses of medical facilities in nursing home residents. Geriatr Gerontol Int. 2016;16(6):770-1.

9. Komiya $H$, Umegaki $H$, Asai A, et al. Factors associated with polypharmacy in elderly home-care patients. Geriatr Gerontol Int. 2018;18(1):33-41.

10. Feng X, Tan X, Riley B, Zheng T, Bias T, Sambamoorthi U. Polypharmacy and multimorbidity among Medicaid enrollees: a multistate analysis. Popul Health Manag. 2018;21(2):123-9.

11. Matsuda T, Iwagami M, Suzuki T, Jin X, Watanabe T, Tamiya N. Correlation between the Barthel Index and care need levels in the Japanese long-term care insurance system. Geriatr Gerontol Int. 2019;19:1186-7.(in press).

12. Moriyama Y, Tamiya N, Kawachi N, Miyairi M. What makes super-aged nations happier? Exploring critical factors of happiness among middle-aged men and women in Japan. World Med Health Policy. 2018;10(1):83-98.

13. Kojima T, Akishita M, Kameyama Y, et al. High risk of adverse drug reactions in elderly patients taking six or more drugs: analysis of inpatient database. Geriatr Gerontol Int. 2012;12(4):761-2.

14. Rosenbaum PR, Rubin DB. The central role of the propensity score in observational studies for causal effects. Biometrika. 1983;70:41-55.

15. Calderón-Larrañaga A, Poblador-Plou B, González-Rubio F, Gimeno-Feliu LA, Abad-Díez JM, Prados-Torres A. Multimorbidity, polypharmacy, referrals, and adverse drug events: are we doing things well? Br J Gen Pract. 2012;62(605): e821-6.

16. Santos-Pérez MI, Fierro I, Salgueiro-Vázquez ME, Gallardo-Lavado MDM, Sáinz-Gil M, Martín-Arias LH. A polypharmacy risk prediction model for elderly patients based on sociodemographic and clinical factors. Int J Clin Pharmacol Ther. 2018:56(12):577-84.

17. Suzuki Y, Akishita M, Arai $\mathrm{H}$, et al. Multiple consultations and polypharmacy of patients attending geriatric outpatient units of university hospitals. Geriatr Gerontol Int. 2006;6:244-7.

18. Carmona-Torres JM, Cobo-Cuenca Al, Recio-Andrade B, Laredo-Aguilera JA, Martins MM, Rodríguez-Borrego MA. Prevalence and factors associated with polypharmacy in the older people: 2006-2014. J Clin Nurs. 2018;27(15-16): 2942-52.

19. The Ministry of Health, Labour and Welfare. Statistics of Medical Care Activities in Public Health Insurance. Available from URL: https://www.mhlw. go.jp/toukei/list/26-19.html (in Japanese).

20. Niikawa $\mathrm{H}$, Okamura T, Ito $\mathrm{K}$, et al. Association between polypharmacy and cognitive impairment in an elderly Japanese population residing in an urban community. Geriatr Gerontol Int. 2017;17(9):1286-93.

\section{Publisher's Note}

Springer Nature remains neutral with regard to jurisdictional claims in published maps and institutional affiliations.

\section{Ready to submit your research? Choose BMC and benefit from:}

- fast, convenient online submission

- thorough peer review by experienced researchers in your field

- rapid publication on acceptance

- support for research data, including large and complex data types

- gold Open Access which fosters wider collaboration and increased citations

- maximum visibility for your research: over $100 \mathrm{M}$ website views per year

At $\mathrm{BMC}$, research is always in progress.

Learn more biomedcentral.com/submissions 